
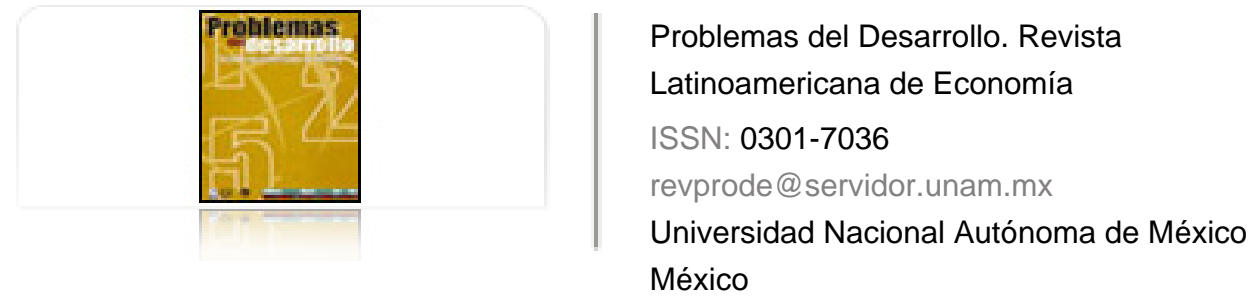

Galindo, Carlos; Delgado, Javier

\title{
LOS ESPACIOS EMERGENTES DE LA DINÁMICA RURAL-URBANA
}

Problemas del Desarrollo. Revista Latinoamericana de Economía, vol. 37, núm. 147, octubre-

diciembre, 2006, pp. 187-216

Universidad Nacional Autónoma de México

Distrito Federal, México

Disponible en: http://www.redalyc.org/articulo.oa?id=11820100008

Cómo citar el artículo

- Número completo

- Más información del artículo

Página de la revista en redalyc.org

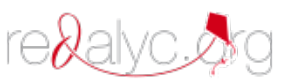

Sistema de Información Científica

Red de Revistas Científicas de América Latina, el Caribe, España y Portugal Proyecto académico sin fines de lucro, desarrollado bajo la iniciativa de acceso abierto 


\title{
LOS ESPACIOS EMERGENTES DE LA DINÁMICA
}

\author{
RURAL-URBANA*
}

\section{Carlos Galindo** Javier Delgado***}

Fecha de recepción:10 de abril de 2006. Fecha de aceptación: 13 de septiembre de 2006.

\section{Resumen}

La multiplicación de efectos socioespaciales derivados de la intensa descentralización regional que se presenta en México desde los años ochenta demanda la búsqueda de opciones teóricas y metodológicas que faciliten la comprensión de la naturaleza y orientación de esos procesos. En este trabajo, se parte del reconocimiento del agotamiento conceptual de los términos urbano y rural, basados en el modelo tradicional de oposición campo-ciudad, para explorar alternativas analíticas basadas en el nuevo paradigma emergente (aún en construcción) de interfase rural-urbana. Así, se presenta una metodología que permite identificar la dinámica rural-urbana en espacios periurbanos, tomando como caso de estudio la corona regional de la ciudad de México.

Palabras clave: interfase rural-urbana, espacio periurbano, rurbanización.

\section{Abstract}

The multiplication of socio-spatial effects derived from the intensive regional decentralization observed in Mexico since the 1980s demands a search for theoretical and methodological options that will facilitate an understanding of the nature and orientation of those processes. In this work, we begin by recognizing the conceptual exhaustion of the terms urban and rural, based on the traditional model of country-city opposition, to explore analytical alternatives based on the new emerging paradigm (still under construction) of rural-urban inter-phase. Thus, a methodology is presented which makes it possible to identify the rural-urban dynamic in peri-urban spaces, taking as a case study the regional periphery of Mexico City.

Key words: rural-urban inter-phase, peri-urban space, urbanization.

* Este trabajo forma parte de la tesis de maestría que Carlos Galindo elabora en el Posgrado en Geografía y también del proyecto CONACYT SEMARNAT 1430 "Interfase rural urbana en la Cuenca Alta del Lerma. Hacia una metodología unificada de ciencias y naturales (CARL) - que se elabora en el Instituto de Geografía, UNAM - bajo la coordinación de Javier Delgado.

*** Tesista y colaborador dentro del citado proyecto. Correo electrónico: mcarlosgp@yahoo.com

* Investigador titular A, Tiempo completo, Definitivo, Instituto de Geografia, UNAM. Correo electrónico: jdelgado@igg.unam.mx 
Résumé

La multiplication des retombées sur l'espace social de l'intense décentralisation régionale effectuée au Mexique depuis les années quatre-vingt, impose la recherche d'options théoriques et méthodologiques qui puissent faciliter la compréhension de la nature et de l'orientation de ces processus. Dans ce travail, il est admis l'épuisement des concepts revêtus par les termes urbain et rural, fondés sur le modèle traditionnel d'opposition entre campagne et ville, et des analyses alternatives sont explorées à partir du nouveau paradigme émergent (encore en construction) d'interface rural-urbain. Il est ainsi présenté une méthodologie qui permet d'identifier la dynamique rural-urbain dans les espaces périurbains, en prenant comme cas d'étude la région périphérique de Mexico.

Mots-cléfs: interface rural-urbain, espace périurbain, ré-urbanisation.

\section{Resumo}

A multiplicação de efeitos sócio-espaciais derivados da intensa descentralização regional que se apresenta no México desde os anos 80, exige a procura de opções teóricas e metodológicas que facilitem a compreensão da natureza e a orientação desses processos. Neste trabalho, parte-se do reconhecimento do esgotamento conceitual dos termos urbano e rural, baseados no modelo tradicional de oposição campo-cidade, para explorar alternativas analiticas baseadas no novo paradigma emergente (ainda em construção) de interfase rural-urbana. Sendo assim, apresenta-se uma metodologia que permite identificar a dinâmica rural-urbana em espaços periurbanos, tomando como caso de estudo a fronteira regional da cidade do México.

Palavras chave: interfase rural-urbana, espaço periurbano, rurbanização. 
Esfuerzos recientes hacia la reconceptuación teórica de los espacios periurbanos

no de los efectos territoriales más notables derivados del proceso de des-
centralización económica — que se presenta en México desde la década de
los ochenta_- es la formación de ámbitos donde lo urbano se asocia con lo rural. El resultado es la formación de espacios complejos, difíciles de analizar con conceptos tradicionales, todavía vigentes en diferentes disciplinas de corte social y, en particular, en la geografía urbana y regional.

Algunas de las investigaciones en las cuales se ha afrontado esa problemática son los trabajos de Ávila (2001 y 2004); Cruz (2002); Ramírez (2003); Sobrino (2003); Torres (2000); Hiernaux (2000); Arias (2005); Banzo (2005) y Delgado (2003). Con perspectivas distintas, en trabajos de carácter más de exploración que concluyentes, han realizado aportes valiosos para el esclarecimiento de esos complejos espacios de contacto rural-urbano.

Ávila (2001:109) analiza la formación de espacios de contacto entre la ciudad y su ámbito regional, que pueden ser identificados como "espacios rururbanos" o "periurbanos". ${ }^{1}$ A partir del reconocimiento de la peculiar dinámica rural-urbana en dichos sitios y del análisis de las transformaciones territoriales que se derivan de ello, señala las principales diferencias en las zonas periurbanas en el caso de Europa y Latinoamérica. ${ }^{2}$ En su ensayo reconoce nociones como espacios del commuting, franja urbano-rural, franja límite, corona periférica que se han propuesto para conceptuarlos. De acuerdo con la idea de la formación periurbana, identifica tres bloques temáticos (espacial, económico y social) pendientes en la agenda investigativa sobre la constitución de los espacios periurbanos en México (ibid.:123-124). ${ }^{3}$ En

1 Por espacio rururbano se entiende la coexistencia e interacción de elementos urbanos y rurales en un mismo territorio, como resultado de la difusión de actividades y población urbana hacia las zonas rurales que le rodean sin que éstas pierdan totalmente sus atributos económicos, sociales o territoriales como sucedía anteriormente con la conurbación. Lo periurbano es un espacio genérico que rodea a cualquier ciudad independientemente de su actividad, función o tipo de ocupación. Cualquier sitio alrededor de la ciudad es periurbano pero no cualquiera es rururbano (Delgado, 2003:28).

2 Las diferencias mencionadas son: a) extensión; $b$ ) nivel de planeación y tipo de ocupación; c) nivel de desarrollo socioespacial; d) homogeneidad o heterogeneidad de los actores sociales presentes.

3 Esta agenda de trabajo se divide en tres bloques principales: 1) espacial: la construcción del periurbano por la expansión urbana y su impacto ambiental en áreas rurales circundantes; 
otro trabajo reciente y con base en una amplia revisión bibliográfica, Ávila (2004) concluye que uno de los efectos de la reestructuración económico-territorial actual es la formación de zonas rururbanas o periurbanas en la periferia de las ciudades que constituyen el escenario de prácticas económicas, sociales, culturales y territoriales asociadas a la agricultura y la industria.

Por otro lado, a partir de la tenencia de la tierra como eje de análisis primordial, Cruz (2002:43) vincula la periferia rural al proceso de urbanización en el caso de la ciudad de México. La presencia de una intensa actividad agrícola periurbana impulsa, según la autora, la formación de espacios en donde coexisten, indistintamente, elementos urbanos y rurales. Además de cuestionar el carácter metropolitano de ciertas delegaciones y municipios no conurbados y ante la dificultad de considerar a esta periferia como rural o urbana, reconoce la posibilidad de caracterizarlos como rururbanos (ibid.:48).

Ramírez (2003), por su parte, hace una amplia revisión de las diferentes categorías de corona regional, zona suburbana o periférica, frontera rural-urbana y espacios yuxtapuestos. En esos originales espacios intermedios o de transición ocurre el encuentro rural-urbano y por ello lo uno y lo otro. Han sido estudiados de forma fragmentada en disciplinas como el urbanismo o la sociología rural y, además, con fuerte sesgo empírico, según la autora. Esos sitios se inscriben en lógicas socioeconómicas y culturales muy distintas a las nociones tradicionales de oposición campo-ciudad y, es importante subrayarlo, adoptan una escala intermedia entre lo urbano y lo regional.

A su vez, desde un enfoque económico-territorial, a partir del análisis de la distribución por municipio de la población y las actividades económicas en la región Centro del país, Sobrino (2003) propone una interesante tipología de municipios diferenciados como metropolitanos, urbanos, rururbanos y rurales. En su opinión, la importancia de los espacios rururbanos de la región Centro radica en su dinámica y función como receptores de una parte importante de las actividades de la zona metropolitana de la ciudad de México.

A partir de la idea de la sustentabilidad de la agricultura urbana (en el caso mexicano) y del desbordamiento territorial de los procesos urbanos y las implicaciones que conlleva, Torres (2000:9-10) propone la región como una escala pertinente de análisis. En su opinión, "lo urbano" ya no se circunscribe a núcleos y periferias suburbanas, sino a una yuxtaposición de elementos urbanos y rurales. Por ello,

2) económico: reestructuración de actividades y territorios periurbanos por las políticas macroeconómicas o locales y cambios en la agricultura como la sustitución o especialización de cultivos; y 3) social: incidencia de la acción de los actores sociales tradicionales y los nuevos agentes rurales y la formación de nuevas redes socioterritoriales en donde los factores culturales son decisivos (Ávila, ibid.). 
propone redefinir los límites geográficos en función de esa yuxtaposición, en reconocimiento de nuevas territorialidades urbano-rurales en donde se articulan mercados de trabajo urbano y rural.

A su vez, para Hiernaux (2000:31-40) se trata de un cambio en el paradigma prevaleciente en las ciencias sociales y cuestiona la pertinencia del binomio urbanorural dentro de la corriente estructural-funcionalista. Es necesario, dice, replantear teóricamente la relación campo-ciudad y analizar las periferias como espacios protagonistas en donde tiene lugar la conformación de nuevas formas de complementariedad entre lo urbano y lo rural que dan lugar a nuevas estructuras espaciales complejas.

En la misma línea de indagación en los "espacios de contacto rural-urbanos", Banzo (2005:209) revisa la cuestión periurbana en Francia y España, desde tres enfoques principales: 1) la percepción de lo periurbano no sólo como territorio, sino como forma de vida; 2) la necesidad de cambiar de escala para analizar la difusión urbana sobre el periurbano, y 3) el desafío que la ciudad difusa representa para las políticas urbanas.

Por su parte, desde la antropología - y reconociendo la existencia de una vieja ruralidad—, Arias (2005) señala que en el proceso de construcción de los espacios urbano-rurales es indiscutible un cambio en los mercados de trabajo rural. Ello se manifiesta en el arribo de la industria maquiladora al campo o en la ocupación, en el sector servicios, de población de origen rural con baja calificación. Son actores rurales y formas de producción nuevos, mecanismos de comercialización y consumo, mismos que tienen un reflejo territorial que sobrepasa el tradicional enfoque campo-ciudad.

Por último, Delgado (2003), apoyándose en el concepto corona regional, sugiere que para el caso de la ciudad de México el proceso de difusión urbana es resultado de diferentes fuerzas como la económica, la social y la propiamente urbana; así, identifica variantes de conexión entre la ciudad y su espacio periurbano circundante.

Por separado, estos trabajos constituyen un esfuerzo inicial por superar el tradicional paradigma de oposición campo-ciudad y, asimismo, son el precedente de la labor de reconceptuación teórica de esos espacios desde el enfoque de interfase rural-urbana que aquí se propone.

\section{Hacia la definición del paradigma de la interfase rural-urbana}

La permanencia de los conceptos tradicionales en diferentes ramas de las ciencias sociales se está convirtiendo en un lastre que frena la labor analítica o al menos la 
constriñe a moldes preestablecidos. Por ello, es prioritaria la búsqueda de opciones teóricas y metodológicas que ayuden a superar esos obstáculos.

En particular, en el caso de la geografía regional, la tradicional dicotomía ruralurbana quizá sea uno de los principales problemas por superar. Desde fines del siglo xx comenzó a gestarse una conformación de ciudad que rebasa el modelo urbanoindustrial como patrón de urbanización dominante. En la actual fase: a) la ciudad ya no puede circunscribirse a una estructura urbana intramuros y, b) los ámbitos otrora rurales se incorporan a la dinámica de las relaciones económicas, políticas y culturales y de comunicación que encabezan los nodos urbanos (Borja y Castells, 1999:11). Hoy es anacrónico seguir haciendo referencia a un mundo rural encasillado en los viejos modelos urbanos y regionales.

Por otra parte, se reconoce un proceso de difusión urbana desde la ciudad principal hacia las ciudades vecinas (medias y pequeñas) que forman una red urbanoregional, que ha superado ampliamente el enfoque anterior de la conurbación (como espacio continuo), y lo sustituye por un modelo de urbanización dispersa (discontinuo) (Dematteis, 1998). Ello también hace necesario la sobreposición de la escala metropolitana con la regional, que combina el enfoque de ciudad-punto con el enfoque de ciudad-área, donde los espacios periurbanos asumen un papel de primer orden, de interfase (Delgado y Ruiz, 2005).

Un breve repaso de los principales supuestos de los modelos urbanos y regionales tradicionales permitirá destacar algunos elementos necesarios para reinterpretar la nueva realidad urbano-regional. En primer lugar, en los modelos en los que se piensa a la ciudad como punto, $a$ ) no consideran el espacio periurbano; $b$ ) no toman en cuenta los intercambios con los actores y áreas periurbanas, y c) son complementarios, pero sólo entre sí.

En efecto, el más conocido y criticado — modelo de círculos concéntricos construido sobre la noción regional "centro-periferia" de Von Thunen pero circunscrito al ámbito urbano y que después sería formalizado por Alonso (1964) como fricción de la distancia - se refiere a la gradual disminución en la localización de vivienda y otros usos económicos conforme se alejan del centro, en el punto donde se maximiza la utilidad de la localización según sus ingresos, lo que afecta significativamente la densidad, precio e intensidad de uso del suelo. Este modelo que hacía referencia a una estructura urbana monocéntrica, hoy es más bien útil para identificar la dinámica de la expansión urbana y predecir la futura (Suárez y Delgado, 2006a). Por el contrario, los dos modelos restantes, el de sectores radiales y, sobre todo, el de núcleos múltiples, fueron concebidos como modelos de morfología urbana para tratar de entender la emergencia de una estructura policéntrica en las ciudades más dinámicas y de la segregación intraurbana que les caracteriza. Esta 

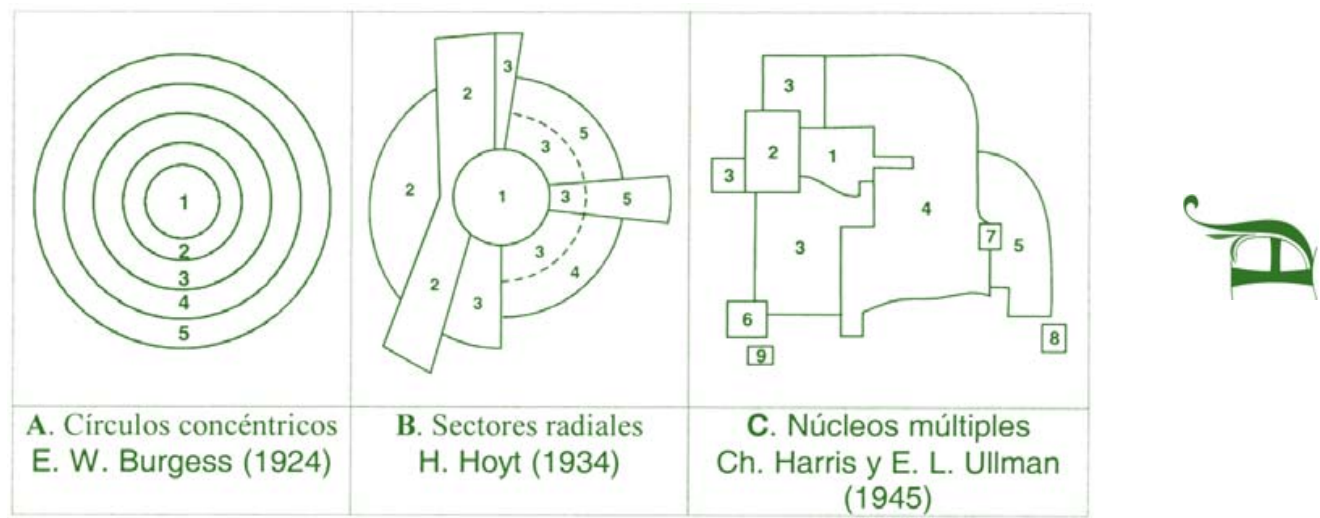

Gráfica 1. Modelos tradicionales en torno de la estructura urbana.

Fuente: De Blij (1981).

sectorización o nucleamiento de la estructura urbana estaría en función de la desigual distribución de los usos del suelo, principalmente para vivienda, de la infraestructura, en particular, de los grandes ejes de comunicación y de la concentración de unidades económicas y empleo, además del tradicional CBD, en nuevos centros en las áreas intermedias (Richardson, 1986; Suárez y Delgado, 2006b). Los tres son complementarios entre sí, pero sólo a escala intraurbana. Los dos primeros modelos excluyen el análisis de los espacios de contacto rural-urbano y sólo el tercer modelo, de núcleos múltiples, incorpora tímidamente la dimensión suburbana como una anticipación sorprendente de la difusión futura (Gráfica 1).

Sólo hasta muy recientemente se ha empezado a explorar la significación de la extensión de la ciudad sobre su espacio periurbano no conurbado, denominado exurbia, en la tradición anglosajona. En este caso, la aglomeración principal ocupa el lugar central y el hinterland se amplía hasta incluir nuevos centros (edge cities en el caso estadounidense, ciudades secundarias medias y pequeñas, en el nuestro) gracias a la construcción de una gran carretera circundante (regional belt) que los comunica entre sí y con la ciudad central. Un caso paradigmático de esos nuevos centros son las plataformas multimodales de transporte, que preferentemente se ubican en las estratégicas intersecciones regionales (Harris, 1997) (Gráfica 2).

Desde otra perspectiva teórica, el modelo posmoderno de estructura urbana de Dear y Flusty (2002:203) pretende un rompimiento epistemológico radical con los anteriores modelos de la Escuela de Chicago mediante la superación de la visión intramuros que los caracteriza. Tomando como caso de estudio a la ciudad de Los Angeles y al contrario de la fuerte constricción del modelo de Harris, sugieren que la nueva localización centro-periferia no sigue ningún orden y se acomoda aleatoriamente, como en un tablero de ajedrez, según la decisión de los actores. Un 


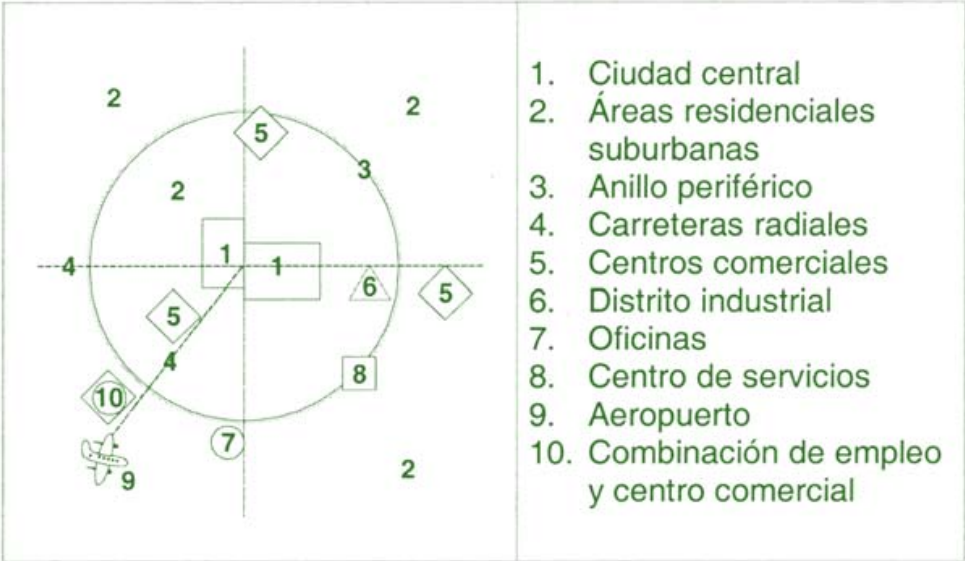

Gráfica 2. Modelo periférico de áreas metropolitanas.

Fuente: elaboración propia con base en Harris (1997).

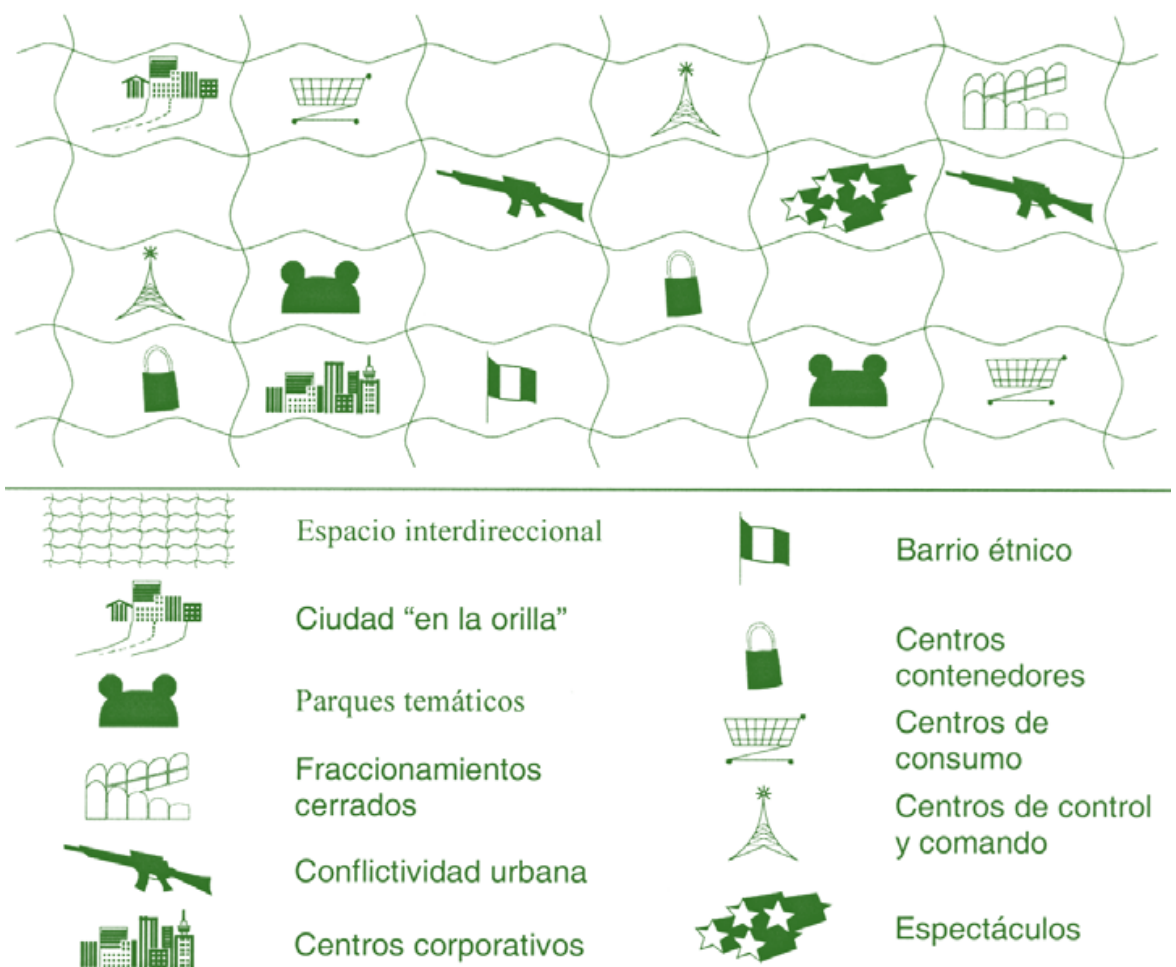

Gráfica 3. Modelo posmoderno de estructura urbana. Fuente: Dear y Flusty (2002:231). 
acierto de este modelo es reconocer que, en el contexto actual de globalización económica, la periferia es quien organiza a la ciudad y no al contrario como sucede en los otros modelos, pero desestima la capacidad de estructuración del territorio de los grandes capitales o de las obras de infraestructura, que no tienen nada de aleatorio (Gráfica 3).

Por su parte, los modelos regionales tradicionales que conciben la ciudad como área se distinguen por: $a$ ) reducir la estructura intraurbana a una dimensión puntual; $b$ ) subestimar el espacio periurbano entre dos núcleos regionales a una categoría de espacio contenedor y, $c$ ) de manera similar a los modelos urbanos, estos enfoques regionales resultan complementarios también, pero sólo entre sí mismos. En ese sentido no existe, hasta hora, un modelo urbano-regional único.

En el caso de la región homogénea, a partir de la influencia que la sociedad ejerce en el medio geográfico-físico, se supone cierto grado de complementariedad entre los elementos físicos y humanos que la integran, dando origen a una región agrícola, pecuaria o industrial. Por su parte, la región nodal o funcional que, a partir de una red jerarquizada de ciudades, delimita un área de influencia, es el modelo más exitoso y todavía vigente, pero subestima la importancia de los espacios intermedios de la red. La región plan consiste en una demarcación administrativa que sirve de referencia espacial para la aplicación de políticas regionales o de ordenamiento territorial (Boudeville, 1961), pero, a diferencia de la dos anteriores, no cuenta con estamento teórico propio y, con frecuencia, no guarda ninguna correspondencia con las otras dos (Gráfica 4).

Ante las insuficiencias de estos modelos regionales, para analizar la conformación regional de ciudad, se han propuesto modelos alternativos entre los que destacan la región flexible y la región geocultural de Giménez.

En la región flexible, Benko y Lipietz (1994) suponen que, a partir del proceso de segmentación de los sistemas productivos y su dispersión territorial, la estructura de las redes urbanas se ha modificado para incorporar ciudades medias y pequeñas, que tienden a formar nuevos espacios subregionales. En función del influjo que la globalización tiene en la dinámica y reestructuración de las regiones, este proceso da resultado espacios o regiones ganadoras y perdedoras, pero no proponen un modelo espacial propiamente dicho.

Por su parte, el planteamiento de región geocultural propone incorporar elementos que no habían sido tomados en consideración en los modelos dominantes (Giménez, 2000). Esos criterios de definición simbólica y material, de acuerdo con su autor, guardan estrecha relación con las particularidades propias del territorio y su vinculación con la sociedad, lo que genera un sentimiento de pertenencia e 


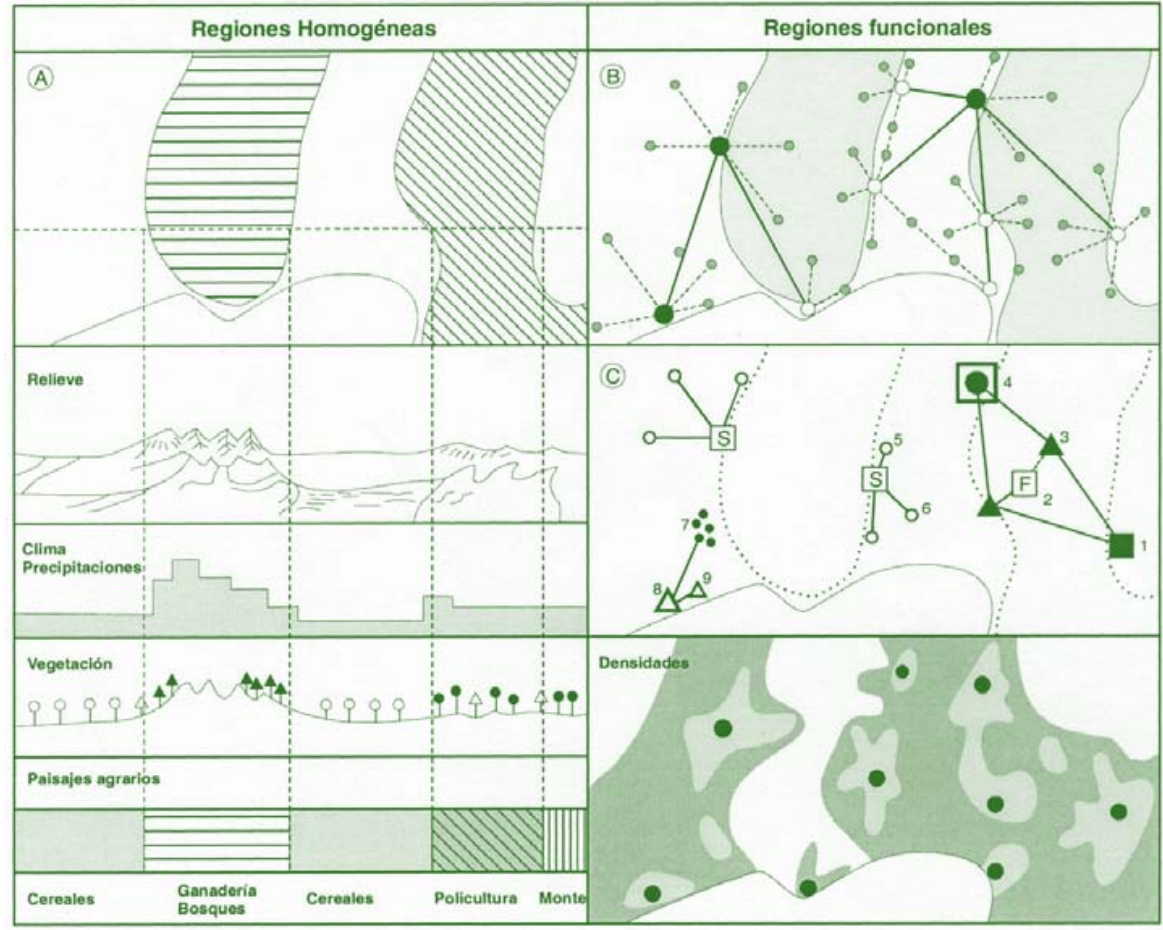

Gráfica 4. Modelos en torno de la estructura regional.

Fuente: Claval (1980:150).

identidad socioterritorial a partir del cual se delimita la región. Tampoco arriba a sugerir un modelo espacial como resultado de lo anterior.

No obstante los esfuerzos teóricos por brindar una alternativa a los modelos regionales tradicionales, se trata de dimensiones estrictamente reflexivas y cualitativas que no se han formalizado en un modelo espacial para comprender la configuración espacio-regional de la ciudad.

Con base en lo anterior, en este trabajo se propone una metodología alterna, acorde al proceso de urbanización difusa donde se articula lo urbano con lo regional. Es necesario pasar de un enfoque bipolar campo-ciudad, a uno donde la bipolaridad se difumine y ocurra un proceso de incorporación a la red urbanoregional. Pasar de los sistemas de ciudades unidas por distintos flujos a una franja periurbana, que deviene un espacio de interfase cuando lo urbano interactúa con lo rural (Gráfica 5). Si bien todo espacio que rodea a la ciudad es, por definición, periurbano, sólo donde existe una interacción rural-urbana hay interfase.

Este nuevo tipo de ruralidad se caracteriza porque los procesos económicos y sociales en el campo son cada vez más complejos y su principal manifestación territorial es la formación de espacios híbridos donde lo rural y lo urbano se ensam- 


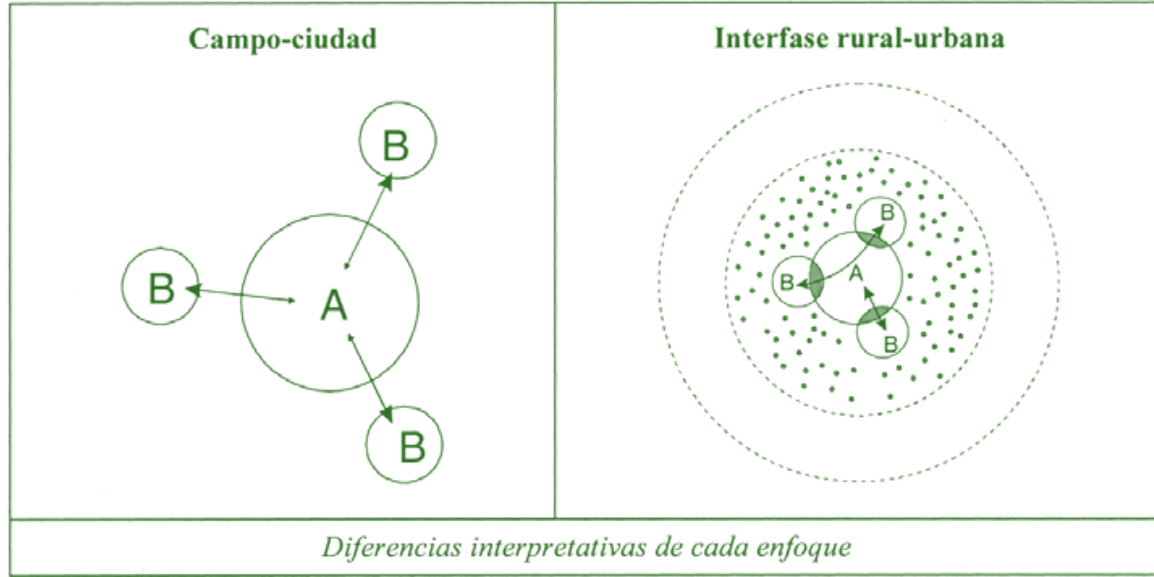

Oposición ......................................................................................... Sobreposición

Dependencia y subordinación ..................................................... Complementariedad e interrelación

Relaciones inerciales ................................................................ Relación dinámica

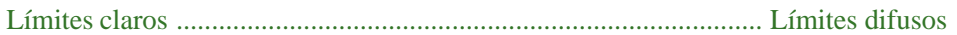

Ubicación puntual .................................................................... Ubicación zonal

Áreas diferenciadas .................................................................... Espacios de interfase

Espacio homogéneo ....................................................................... Heterogeneidad espacial

Gráfica 5. De la oposición campo-ciudad a la interfase rural-urbana.

Fuente: elaboración propia.

blan y son difíciles de identificar, analizar y contextualizar con los paradigmas tradicionales (Delgado et al., 2003). Ahora bien, ¿es posible reconocer la dinámica rural-urbana en espacios periurbanos? $\mathrm{Y}$, sobre todo, en términos de modelos espaciales, ¿cómo delimitar territorialmente esos espacios?

\section{Hacia una metodología para identificar los espacios rural-urbanos}

\section{Los antecedentes}

Desde la década de los setenta, diversos autores han intentado medir esta difusión en el caso de la ciudad de México y la región Centro, que al parecer representa la formación espacial de este tipo más extendida en el país. Esos esfuerzos se han guiado por el enfoque predominante de región nodal o funcional, esto es, de una concepción de la ciudad como punto.

El primero en llamar la atención sobre el tema, si bien no lo formalizó en un modelo, fue Flores (1961) al sugerir, con más ironía que ciencia, llamar MexСиеPиePaToTla al sistema territorial resultante, utilizando las primeras letras del nombre de las ciudades involucradas en la megalópolis emergente. En efecto, varios años después de Flores, Unikel et al. (1978) sugirieron la formación de una megalópolis con base en los flujos de transporte entre las ciudades de la región Centro. 
En esta línea de investigación —-flujos en lugar de áreas—, Graizbord y Gallardo (1986) realizaron el primer análisis formal de esa red urbano-regional mediante un modelo gravitacional de flujos telefónicos, cuando esos datos todavía eran públicos, pues a partir de la privatización de TELMEX se consideran confidenciales y son inaccesibles para su estudio. Poco después, Kunz y Hernández (1991) realizaron un ejercicio similar que mostró las interrelaciones de la región Centro con las regiones de Occidente, Golfo y norte de Guerrero. Posteriormente, Garza (1992) retomó el criterio de área para formar una megalópolis, al proponer el traslape funcional de las áreas de influencia de las distintas metrópolis involucradas, independientemente del valor de aquellos enlaces funcionales. Visto así, la megalópolis se reduce a las ciudades de México y Toluca, cuyo traslape habría ocurrido en algún momento de la década de los ochenta. ${ }^{4}$ Más recientemente, Negrete (1999) ha contribuido a este debate silencioso al incluir el análisis de las migraciones hacia y dentro de la región Centro como una poderosa fuerza que estructura el territorio.

Después de esos ejercicios pioneros, se han realizado al menos dos más de éstos, formales, que comprueban de forma definitiva la existencia y amplitud de una franja periurbana alrededor de la ciudad de México, nuevamente, desde el enfoque de área. Delgado et al. (1999) sistematizaron, mediante un índice de componentes principales, cinco variables para identificar una franja de unos 100-120 kilómetros alrededor de la ciudad de México a la que llamaron "corona regional". Posteriormente, Aguilar (2002), con un método similar y con énfasis más en el empleo y la formación de subcentros, llegó a una delimitación parecida, un poco menos extensa, a la que llamó "periferias expandidas".

Una debilidad inherente a los ejercicios anteriores es que sobrevaloran la dimensión urbana, asumiendo, de forma implícita, que lo rural o sus remanentes se subsumen en esa área. En este trabajo se intenta explorar el potencial de los indicadores de la rurbanización en la conformación de la corona regional de la ciudad de México. El resultado más relevante de este ejercicio es que, ambas mediciones —el de la difusión urbana y el de la nueva ruralidad de los espacios periurbanos-coexisten funcionalmente en tiempo y espacio, ajustándose a las conjeturas y reflexiones teóricas revisadas en la primera parte.

\section{Una metodología propia para identificar lo rururbano}

De acuerdo con la revisión de la literatura, los dos aspectos principales que caracterizan a los nuevos espacios de la ruralidad son: $a$ ) su fuerte dinámica (entendida no

4 Para una discusión de las diferentes acepciones de megalópolis y una definición de corona regional, véase Delgado (2003). 
sólo como velocidad de cambio (tasa) sino como el cambio estructural que experimenta un ambiente rural al convertirse en urbano), y b) el comportamiento de ciertos procesos típicamente rurales: la dispersión, la intensidad de uso de la tierra agrícola, la industrialización rural y la migración, que comparten con las áreas urbanas, pero que, en esta etapa, alcanza una relevancia antes sólo presente en lugares centrales, urbanos.

Por supuesto, no son éstos los únicos factores de la nueva ruralidad. ${ }^{5}$ Para este ejercicio únicamente se eligieron indicadores estadísticos, cartografiables y disponibles para todos los municipios y para los cuales se contara, al menos, con datos de dos fechas distintas. El enfoque elegido no excluye otras formas de aproximarse al objeto de estudio, como la influencia de los cambios cualitativos de la fuerza de trabajo agrícola, del comportamiento de la familias y actores rururbanos, los cambios de políticas y estrategias productivas accesibles únicamente mediante encuestas locales o muestreo en campo. De hecho, se espera que la identificación de los espacios emergentes aquí propuesta sirva de guía para otros trabajos en escala local y con base en procesos sociopolíticos específicos, difíciles de operar en la escala regional del presente ensayo.

La metodología propuesta consiste, primero, en estimar la dinámica del proceso mediante el cambio de categoría rural-urbana de los municipios de la región, y segundo, en medir la intensidad del cambio con una síntesis factorial de los cuatro indicadores arriba mencionados. Como tercer paso se obtiene una aproximación a la interfase rural-urbana, al cruzar los valores de ambas mediciones en una matriz de doble entrada, con el cambio de categoría en la primera columna y los rangos del índice de rurbanización en las columnas restantes (véase Cuadro 1).

Veamos cada uno de los pasos por separado, antes de analizar la espacialidad de los resultados.

\section{Cambio de categoría rural-urbana en la región Centro}

Este cambio se identifica de acuerdo con la clasificación de Unikel et al. (1978:118) de cuatro categorías de localidades en función de la población total que reside en ellas: $a$ ) rural, menos de cinco mil habitantes; $b$ ) mixta-rural, entre cinco y diez mil; c) mixta-urbana, entre 10 y 15 mil, y $d$ ) urbana, más de 15 mil personas.

$5 \quad$ Otra acepción de nueva ruralidad, presentada por Shejtman y Berdegué (2003), desarrollo territorial rural, también tiene un componente territorial importante, pero con el concepto de "distrito industrial" marshaliano, con base en las características productivas de los ambientes locales. Es factible que alguno de los espacios rururbanos que aquí se plantean pudiesen constituir distritos industriales o cuasi distritos, pero esa discusión rebasa los alcances del presente trabajo. 
Cuadro 1

Identificación de la interfase rural-urbana*

\begin{tabular}{|c|c|c|c|c|c|}
\hline \multirow[b]{2}{*}{$\begin{array}{c}\text { Cambio de categoría } \\
\text { rural-urbana (1970-2000) }\end{array}$} & \multicolumn{5}{|c|}{$\begin{array}{l}\quad \text { Índice de rurbanización } \\
\text { a) Dispersión rural } \\
\text { b) Intensidad de uso de la tierra agrícola } \\
\text { c) Industrialización rural } \\
\text { d) Migración indirecta }\end{array}$} \\
\hline & $\begin{array}{l}\text { Muy bajo } \\
\text { (1) }\end{array}$ & $\begin{array}{l}\text { Bajo } \\
\text { (2) }\end{array}$ & $\begin{array}{l}\text { Medio } \\
\text { (3) }\end{array}$ & $\begin{array}{r}\text { Alto } \\
(4)\end{array}$ & $\begin{array}{l}\text { Muy alto } \\
\text { (5) }\end{array}$ \\
\hline \multicolumn{6}{|l|}{ 1. Rural-mixto rural } \\
\hline y mixto rural-mixto urbano & 1 & 1 & 2 & 2 & 3 \\
\hline 2. Rural-mixto urbano & 1 & 2 & 3 & 3 & 3 \\
\hline 3. Mixto urbano-urbano & 2 & 3 & 3 & 4 & 4 \\
\hline 4. Mixto rural-urbano & 2 & 3 & 4 & 4 & 5 \\
\hline 5. Rural-urbano & 3 & 3 & 4 & 5 & 5 \\
\hline
\end{tabular}

* Se trata de las categorías propuestas por Unikel et al. Sin embargo, se agrupan aquí las categorías rural-mixto rural y mixto rural-mixto urbano por ser las de cambio más bajo; y se excluyeron del análisis de componentes los 34 municipios que mantuvieron una categoría urbana entre 1970 y 2000, para que no influyeran estadística ni espacialmente en el resultado (los restantes 498 municipios de la región Centro sí fueron incluidos).

Fuente: elaboración propia.

Para clasificar un municipio como urbano debe contar con al menos una localidad de 15 mil habitantes o más, y lo mismo para los otros rangos. El periodo analizado oscila entre 1970 y 2000, cuando se registraron los cambios fundamentales en el patrón de urbanización del país (Sobrino, 1996). Los resultados arrojan nueve categorías, seis en los umbrales iniciales más tres que no cambiaron de categoría. Este primer resultado muestra una dinámica intensa, pues si bien un poco más de la mitad de los municipios (que involucra $62 \%$ de la población) conservó la categoría que ya tenía en 1970, en el otro extremo, fueron más de 12 millones de habitantes rurales (en 234 municipios) que pasaron a una clasificación intermedia o urbana (Cuadro 2). ${ }^{6}$

Cabe subrayar el caso de las categorías mixtas e incluso la rural, que pasaron directamente a urbanas, pues en 2000 absorbieron 8.9 millones de pobladores (74\% del total del cambio). Este grupo duplicó su importancia porcentual (de 14\% a $28 \%$ ) con tasas de dos y tres veces más el promedio regional. Los segmentos que no cambiaron de sitial están por debajo de ese promedio. Es decir, las categorías intermedias definieron el comportamiento regional, o dicho de otra forma, la periferia, o más exactamente, el periurbano reestructura al conjunto regional.

$6 \quad$ En el enfoque convencional de la urbanización del campo esa fuerte dinámica rural urbana se interpreta como una clara tendencia hacia la urbanización per se. En este ensayo se asume que no todos esos espacios de articulación entre lo urbano y lo rural deban, necesariamente, terminar siendo urbanos. 
Cuadro 2

Cambio de categoría rural-urbana por municipio en la región Centro, 1970-2000

\begin{tabular}{|c|c|c|c|c|c|c|}
\hline \multirow{2}{*}{$\begin{array}{l}\text { Categorías de } \\
\text { municipios }\end{array}$} & \multirow{2}{*}{$\begin{array}{c}\text { Número de } \\
\text { municipios } \\
2000\end{array}$} & \multicolumn{4}{|c|}{$\begin{array}{llllllll}P o & b & l & a & c & i & o & n \\
1970 & & & & & & & \\
1900 & 0\end{array}$} & \multirow{2}{*}{$\begin{array}{c}\text { Tasa de } \\
\text { crecimiento } \\
1970-2000\end{array}$} \\
\hline & & Total & $\%$ & Total & $\%$ & \\
\hline Rural a mixto-rural & 100 & 964947 & 6.2 & 1979181 & 6.3 & 2.42 \\
\hline Mixto-rural a mixto-urbano & 19 & 257447 & 1.7 & 485486 & 1.5 & 2.14 \\
\hline Rural a mixto-urbano & 23 & 292218 & 1.9 & 675871 & 2.1 & 2.83 \\
\hline Mixto-urbano a urbano & 21 & 722304 & 4.7 & 2399610 & 7.6 & 4.08 \\
\hline Mixto-rural a urbano & 50 & 1141632 & 7.4 & 4675122 & 14.8 & 4.81 \\
\hline Rural a urbano & 21 & 308190 & 2.0 & 1898627 & 6.0 & 6.25 \\
\hline Subtotal & 234 & 3686738 & 23.9 & 12113897 & 38.4 & 4.05 \\
\hline Permanece rural & 255 & 1792301 & 11.6 & 2867501 & 9.1 & 1.58 \\
\hline Permanece mixto-rural & 9 & 145682 & 0.9 & 298870 & 0.9 & 2.42 \\
\hline Permanece urbano & 34 & 9821457 & 63.6 & 16251876 & 51.5 & 1.69 \\
\hline Subtotal & 298 & 11759440 & 76.1 & 19418247 & 61.6 & 1.69 \\
\hline Total región Centro & 532 & 15446178 & 100.0 & 31532144 & 100.0 & 2.41 \\
\hline
\end{tabular}

Fuente: cálculos propios sobre la base de: SIC, 1972; INEGI, 2001.

A pesar de lo notable del cambio, cuando se analiza su localización espacial es aún más clara su vinculación con la difusión urbana, lo que será útil para encontrar a la población y los municipios rururbanos que estamos buscando. Para ello, se reagruparon los anteriores resultados en tres ámbitos subregionales: dentro del núcleo central de la zona metropolitana de la ciudad de México (ZMCM), en la corona regional o en la periferia regional, de acuerdo con la estructura propuesta por Delgado et al. (1999).

Así, el peso de la ciudad de México vuelve a aparecer, pues su zona metropolitana concentró en 2000 a 5.8 millones de pobladores que cambiaron de status (en 49 municipios), pero destaca ampliamente el papel emergente de la corona regional al concentrar 4.4 millones en 125 municipios contra sólo 1.8 millones y 60 municipios en la periferia regional. Este resultado confirma que la dinámica del cambio rural-urbano se localiza principalmente ( $84 \%$ del total) en el ámbito metropolitano de la ciudad de México y su corona regional (véase Cuadro 3). Falta contrastar esa dinámica con la intensidad de los indicadores socioeconómicos.

\section{Índice de rurbanización (IRUR)}

Los indicadores que se consideraron representativos de los cambios socioeconómicos asociados al proceso de difusión urbana son: $a$ ) el índice de dispersión rural o índice de Demangeon ajustado; $b$ ) la intensidad de uso de la tierra agrícola; $c$ ) la industrialización rural, y $d$ ) la migración indirecta.

Además de la facilidad para obtener los datos y de su construcción estadística, se seleccionaron los indicadores más utilizados en la literatura especializada como 
CARLOS GALINDO Y JAVIER DELGADO

Cuadro 3

Localización de la dinámica rural-urbana en la región Centro, 2000

\begin{tabular}{|c|c|c|c|c|c|c|c|c|}
\hline & \multicolumn{6}{|c|}{ 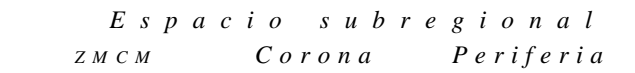 } & \multicolumn{2}{|c|}{$\begin{array}{l}\text { Total región } \\
\qquad \text { Centro }\end{array}$} \\
\hline & Municipios & s Población & Municipios & Población & Municipios & s Población & Municipios & Población \\
\hline Rural a mixto-rural & 10 & 254618 & 59 & 1014707 & 31 & 709856 & 100 & 1979181 \\
\hline Mixto-rural a mixto-urbano & 3 & 59565 & 9 & 258747 & 7 & 167174 & 19 & 485486 \\
\hline Rural a mixto-urbano & 4 & 92513 & 11 & 361104 & 8 & 222254 & 23 & 675871 \\
\hline Mixto-urbano a urbano & 7 & 1492429 & 13 & 837017 & 1 & 70164 & 21 & 2399610 \\
\hline Mixto-rural a urbano & 16 & 2709273 & 24 & 1451061 & 10 & 514788 & 50 & 4675122 \\
\hline Rural a urbano & 9 & 1199699 & 9 & 523870 & 3 & 175058 & 21 & 1898627 \\
\hline Subtotal & 49 & 5808097 & 125 & 4446506 & 60 & 1859294 & 234 & 12113897 \\
\hline Permanece rural & 8 & 78024 & 46 & 434136 & 201 & 2355341 & 255 & 2867501 \\
\hline Permanece mixto-rural & & & 7 & 250033 & 2 & 48837 & 9 & 298870 \\
\hline Permanece urbano & 18 & 12510556 & 12 & 3279630 & 4 & 461690 & 34 & 16251876 \\
\hline Subtotal & 26 & 12588580 & 65 & 3963799 & 207 & 2865868 & 298 & 19418247 \\
\hline Total región Centro 2000 & 75 & 18396677 & 190 & 8410305 & 267 & 4725162 & 532 & 31532144 \\
\hline
\end{tabular}

Fuente: cálculos propios sobre la base de INEGI, 2001.

representativos de la dinámica rururbana. Representan algunos de los rasgos característicos de la nueva ruralidad, como son la alta dispersión sobre el territorio, una creciente pluriactividad económica, el desplazamiento de la industria hacia sitios no urbanos y una alta inmigración en municipios ya no solamente urbanos, sino incluso cada vez más rurales, pero cercanos a una ciudad.

El índice de dispersión rural o índice de Demangeon original (Gutiérrez Puebla, 1992, citado en Palacio et al., 2004), concebido como la proporción de la población rural dispersa respecto de la total municipal, fue ajustado en función de la distancia a la zona metropolitana más próxima para identificar, con mayor certeza, los municipios de mayor dispersión, ya que esa distancia afecta de forma distinta dos municipios a pesar de que ambos tengan el mismo valor del índice.

Éste se calculó de la siguiente forma:

$$
\left.\mathrm{D}=\left(\left(\mathrm{E}^{*} \mathrm{~T}\right) / \mathrm{P}\right) / \mathrm{d}\right)
$$

en la cual $E$ es la población del municipio que reside en localidades de menos de cinco mil habitantes; $T$ el número de localidades en el municipio de menos de cinco mil habitantes; $P$ la población total del municipio; y $d$ la distancia por carretera desde la delegación o cabecera municipal a la zona metropolitana más próxima. ${ }^{7}$

7 Con excepción de la ZMCM, cuya delimitación corresponde a la del Programa (1996), la de las cinco zonas metropolitanas restantes (en orden alfabético: Cuernavaca, Pachuca, Puebla, Tlaxcala y Toluca) hacia las que se calculó la distancia por carretera desde las delegaciones 
El indicador de intensidad de uso de la tierra agrícola, asumido como la proporción de superficie de labor bajo riego de la superficie de labor total, se incluyó por la importancia que tienen las áreas de riego en el proceso de estructuración del territorio. La tecnificación del proceso productivo, así como la utilización de insumos (irrigación, fertilizantes, maquinaria, crédito, entre otros), permite reconocer las áreas de empleo agrícola. Dicha intensidad se calculó con base en la fórmula:

\section{$\mathrm{I}=\mathrm{Sr} / \mathrm{St}$}

en la cual $S r$ es la superficie (en hectáreas) de labor bajo riego del municipio; y $S t$ la superficie (en hectáreas) de labor total del municipio.

Por su parte, la industrialización rural, concebida como las áreas rurales que presentan un cociente de localización industrial, de acuerdo con Delgado (2003a: 103-106), es un fenómeno reciente y aún poco estudiado en México. Se relaciona con el proceso de dispersión regional de la industria que comienza a adquirir una magnitud y extensión territorial importantes, mediante la relocalización de la industria en municipios rurales, en proceso de transición rural-urbana e incluso en aquellos que, de 1970 a 2000, se consolidaron como urbanos (Sobrino, 2003).

En la región Centro del país, los subsectores manufactureros identificados como los más dinámicos, en función de la tasa de crecimiento de su población ocupada, son cuatro: $31,32,33$ y 34 . Coincidentemente, esos sectores se relacionan con actividades agropecuarias o agroindustriales (Cuadro 3). ${ }^{8}$

El procedimiento para identificar la industrialización rural por municipio consistió en el cálculo de un cociente de localización industrial mediante la fórmula:

$$
\mathrm{C}=(\mathrm{Pms} / \mathrm{Pm}) /(\operatorname{Prs} / \mathrm{Pr})
$$

en la cual Pms es la población municipal ocupada en los subsectores 31, 32, 33 y 34, y Pm la población total del municipio ocupada en el sector 3 de actividad; Prs es la población ocupada en los subsectores $31,32,33$ y 34 pero a escala regional, y $\operatorname{Pr}$ la población total de la región ocupada en el sector 3.

Respecto de la migración indirecta, como la proporción de población asociada a movimientos de inmigración o emigración. Se considera que las nuevas tendencias migratorias están condicionadas por la dispersión regional del empleo, lo que implica

y cabeceras municipales, se tomó de Sobrino (2003a). Las seis zonas metropolitanas consideradas son capitales estatales y registran una población superior a 250 mil habitantes.

8 Los subsectores identificados también tuvieron alto dinamismo en la escala nacional, por lo que es plausible la hipótesis de que la industrialización del campo no se restringe únicamente a la región Centro del país. 


\section{CARLOS GALINDO Y JAVIER DELGADO}

204

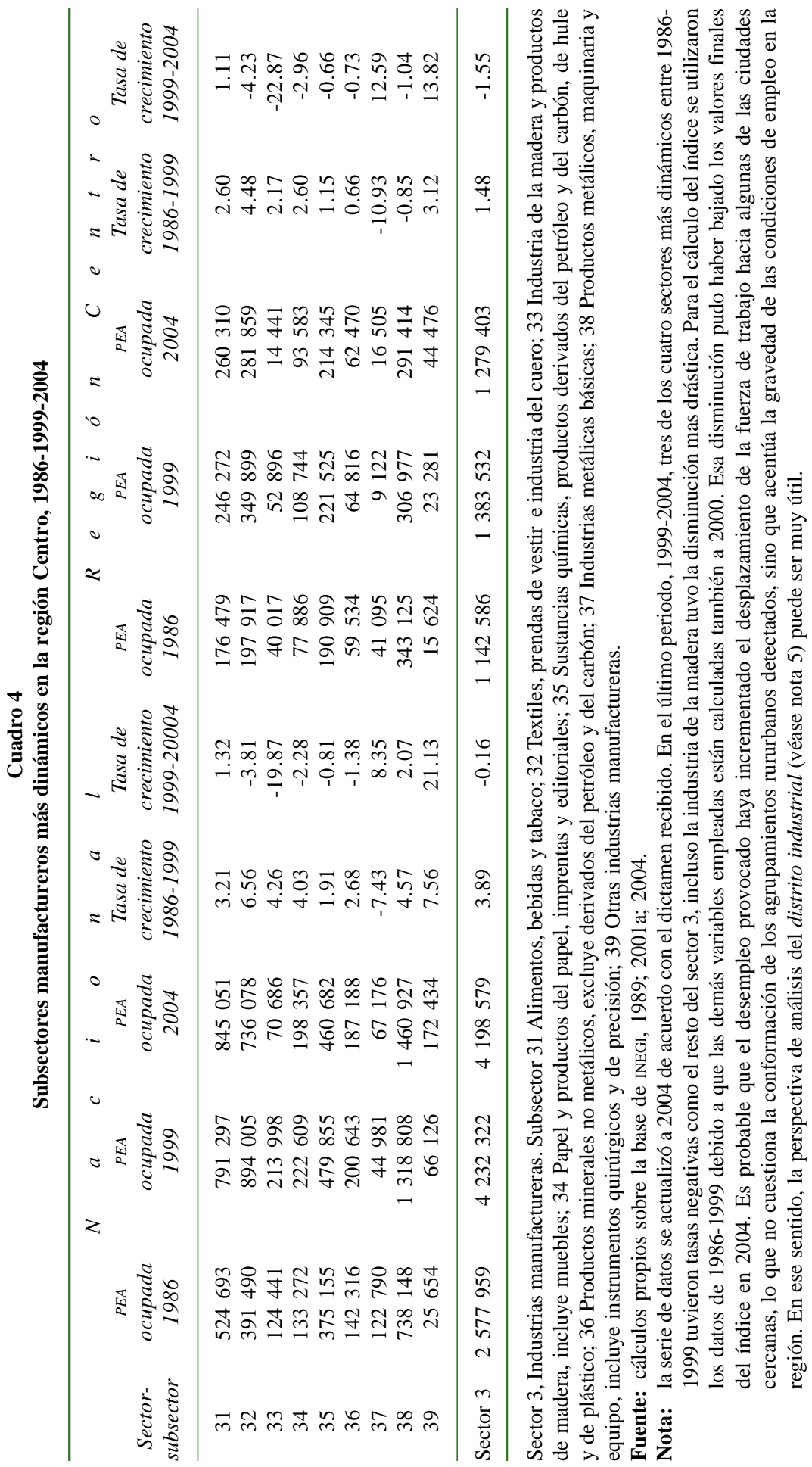


cambios en la preferencia de los migrantes para establecer su residencia o condiciona el desplazamiento de la fuerza de trabajo que es indispensable para las empresas.

Se recurrió a una medición indirecta de la migración, debido a que el registro censal excluye la migración intramunicipal que, en esta fase, se incrementa. La migración indirecta se calculó tomando como base la tasa de crecimiento nacional 1970-2000, como equivalente de la tasa natural debido a la falta de datos para todos los municipios. Cuando la población final obtenida es superior a la censal (real), se interpretó como inmigración, si es menor, como emigración (Camposortega, 1992).

Dentro de esa perspectiva, se advierte un cambio en los patrones migratorios en diferentes países, tanto en desarrollo como en vías de desarrollo y en donde las ciudades de alta primacía han dejado de ser los lugares preferentes de atracción y las ciudades medias y pequeñas se han convertido en los receptores de los flujos migratorios (Vining y Pallone, 1982; Cochrane y Vining, 1988).

Como se puede apreciar, la selección de estos indicadores asumió como criterio prioritario el nivel de representatividad de cada indicador en general y del conjunto en particular, para identificar aquellos territorios en los que el proceso de difusión tiene mayor impacto y, por tanto, donde el proceso de rurbanización está más adelantado (Cuadro 5).

Los resultados de estos indicadores, por separado, prefiguran la amplitud del periurbano. Los municipios con los valores más bajos del índice de dispersión, esto es, zonas de concentración rural, albergan una alta proporción de población. Un poco más de seis millones de pobladores rurales habitan en municipios que tienen de media a muy alta intensidad de tierra agrícola de riego. Por su parte, casi dos millones de personas habitan en 99 municipios con alto y muy alto índice de industrialización rural.

Cuadro 5 Síntesis de los indicadores para la construcción del IRUR, 2000*

\begin{tabular}{|c|c|c|c|c|c|c|c|c|}
\hline \multirow[b]{2}{*}{ Rangos } & \multicolumn{2}{|c|}{$\begin{array}{l}\text { Índice de dispersión } \\
\text { rural (ajustado) }\end{array}$} & \multicolumn{2}{|c|}{$\begin{array}{l}\text { Intensidad de uso } \\
\text { de la tierra agrícola }\end{array}$} & \multicolumn{2}{|c|}{ Migración indirecta } & \multicolumn{2}{|c|}{$\begin{array}{c}\text { Industrialización } \\
\text { rural }\end{array}$} \\
\hline & Municipios & Población & Municipios & Población & Municipios & Población & & Población \\
\hline Muy bajo & 91 & 5588897 & 71 & 3160417 & & & 26 & 1472496 \\
\hline Bajo & 57 & 2330347 & 46 & 2336515 & 7 & 129509 & 53 & 4677317 \\
\hline Medio & 50 & 2399933 & 50 & 2737612 & 48 & 1291552 & 56 & 3082391 \\
\hline Alto & 25 & 1239272 & 37 & 2691251 & 126 & 4457521 & 60 & 1893377 \\
\hline Muy alto & 11 & 555448 & 30 & 1188102 & 53 & 6235315 & 39 & 988316 \\
\hline Región Centro & 234 & 12113897 & & & & & & \\
\hline
\end{tabular}

Fuente: cálculos propios sobre la base de INEGI, 2001.

* La serie estadística de cada uno de los indicadores fue dividida en cinco rangos mediante el método de estratificación de Jenk (Natural Break). 
CARLOS GALINDO Y JAVIER DELGADO

Cuadro 6

Porcentaje de la varianza explicada

\begin{tabular}{|c|c|c|c|c|c|c|}
\hline \multirow[b]{2}{*}{ Componente } & \multicolumn{3}{|c|}{$\begin{array}{c}\text { Autovalores iniciales } \\
\% \text { dela }\end{array}$} & \multicolumn{3}{|c|}{$\begin{array}{c}\text { Suma de las saturaciones al } \\
\text { cuadrado de la extracción } \\
\text { \% de la }\end{array}$} \\
\hline & Total & varianza & $\%$ acumulado & Total & varianza & $\%$ acumulado \\
\hline 1 & 1.417 & 35.428 & 35.428 & 1.417 & 35.428 & 35.428 \\
\hline 2 & 1.013 & 25.316 & 60.743 & 1.013 & 25.316 & 60.743 \\
\hline 3 & 0.829 & 20.733 & 81.476 & 0.829 & 20.733 & 81.476 \\
\hline 4 & 0.741 & 18.524 & 100.000 & 0.741 & 18.524 & 100.000 \\
\hline
\end{tabular}

Método de extracción: análisis de componentes principales.

El siguiente paso metodológico fue sintetizar los valores de los cuatro indicadores mediante un análisis multivariado de componentes principales que muestra una varianza explicada de $100 \%$ en cuatro de éstos en igual número de variables, lo cual se interpretó como un alto valor de resumen estadístico y la ratificación de la selección de variables (Cuadro 6). ${ }^{9}$

Por su parte, los valores obtenidos de la matriz de correlación de componentes también permiten constatar la pertinencia de los indicadores, ya que el primer componente muestra la variación común entre las cuatro variables seleccionadas y su comportamiento es el esperado de acuerdo con el viejo paradigma campo-ciudad: a mayor proporción de uso de suelo agrícola (y distancia al centro), disminuye la industrialización y aumenta la expulsión de población. Sin embargo, este primer componente explica sólo 35\% de la variación común entre los indicadores, lo que significa que el restante $65 \%$ de aquella no se comporta de acuerdo con aquel paradigma (Cuadro 7).

Por el contrario, en el segundo y tercer componente, la intensidad agrícola y la industrialización rural varían conjuntamente en la misma dirección. En el segundo componente, a medida que la intensidad de uso de suelo agrícola disminuye, también lo hace la industrialización rural, pero la dispersión aumenta. En el caso del tercer componente, que a medida que la intensidad de uso de suelo agrícola aumenta, también lo hace la industrialización rural. Cabe destacar, en el caso del tercer componente la migración disminuye a medida que las otras variables aumentan. En síntesis, estos dos componentes explican $45 \%$ de la varianza y muestran modelos

9 Se optó por el método de análisis multivariado de componentes principales porque: 1) conden sa estadísticamente las variables consideradas; 2) determina el peso específico de cada variable seleccionada mediante el valor de la correlación, y 3) integra en nuevas variables la variación conjunta de diferentes indicadores por medio de los cuales se establece la variación total. Pero para el propósito del artículo es importante que explique por qué se utiliza este análisis. 
Cuadro 7

Matriz de correlación de componentes del Índice de Rurbanización*

\begin{tabular}{|c|c|c|c|c|}
\hline Indicador & 1 & $m_{2} p$ & $\begin{array}{ll}e & n \\
3\end{array}$ & 4 \\
\hline Índice de dispersión rural (ajustado) & 0.039 & 0.974 & 0.189 & 0.119 \\
\hline Intensidad de uso de la tierra agrícola & 0.629 & -0.210 & 0.742 & 0.099 \\
\hline Industrialización rural & -0.721 & -0.140 & 0.230 & 0.638 \\
\hline Migración indirecta & 0.707 & -0.010 & -0.436 & 0.556 \\
\hline
\end{tabular}

Método de extracción: Análisis de Componentes Principales.

* Aunque uno de los propósitos del método de análisis de componentes principales es reducir el número de variables necesarias, encontrando la variación común, en el caso de estas cuatro variables esa variación no es evidente, por lo cual el número de componentes con una proporción significativa de variación explicada y las variables es el mismo.

que no se ajustan a los planteamientos del viejo paradigma de oposición campociudad y puede ser la prueba de que, en efecto, la rurbanización es factible.

También es preciso mencionar que las correlaciones de intensidad agrícola e industrialización rural no son muy altas en los componentes 2 y 3 , lo cual indica lo incipiente del proceso. Por el contrario, el cuarto componente que explica $18 \%$ de la varianza muestra un modelo aún más anómalo, al registrar todos los componentes positivos.

Posteriormente, la serie estadística del primer componente fue dividida en cinco rangos mediante el método de estratificación de Jenk (natural break). ${ }^{10}$ Esta clasificación cuantitativa sirvió para diferenciar cualitativamente el índice de rurbanización por municipio: mиy bajo, bajo, medio, alto у muy alto.

Por último, se elaboró la matriz de doble entrada como se indicó anteriormente, con la dinámica en la primera columna y los rangos del índice de rurbanización en las restantes columnas. El mayor valor (5) se asignó a los municipios que alcanzaron el cambio más fuerte de categoría rural-urbana y al mismo tiempo registraron un muy alto índice de rurbanización. En el otro extremo, se asignó el menor valor (1) a aquellos municipios con el más bajo cambio de categoría y también obtuvieron bajos valores del índice de rurbanización. Los valores intermedios (2 a 4) se distribuyeron en los rangos intermedios en ambos lados de la matriz. El resultado obtenido de la matriz fue cartografiado y presenta los siguientes características:

a) los municipios que alcanzaron alto y muy alto dinamismo y difusión predominan al interior de la corona regional $\mathrm{y}$, definitivamente, forman los periurbanos

10 Se recurrió a este método (Cromley, 1996) porque establece los cortes entre los diferentes estratos a partir de la minimización de la varianza dentro de cada uno de ellos, lo cual arroja rangos bien diferenciados entre sí. Una alternativa es la estratificación por quintiles, pero ello conduce a formar grupos homogéneos en cuanto a su número, pero no en términos de la variación de la variable observada. 


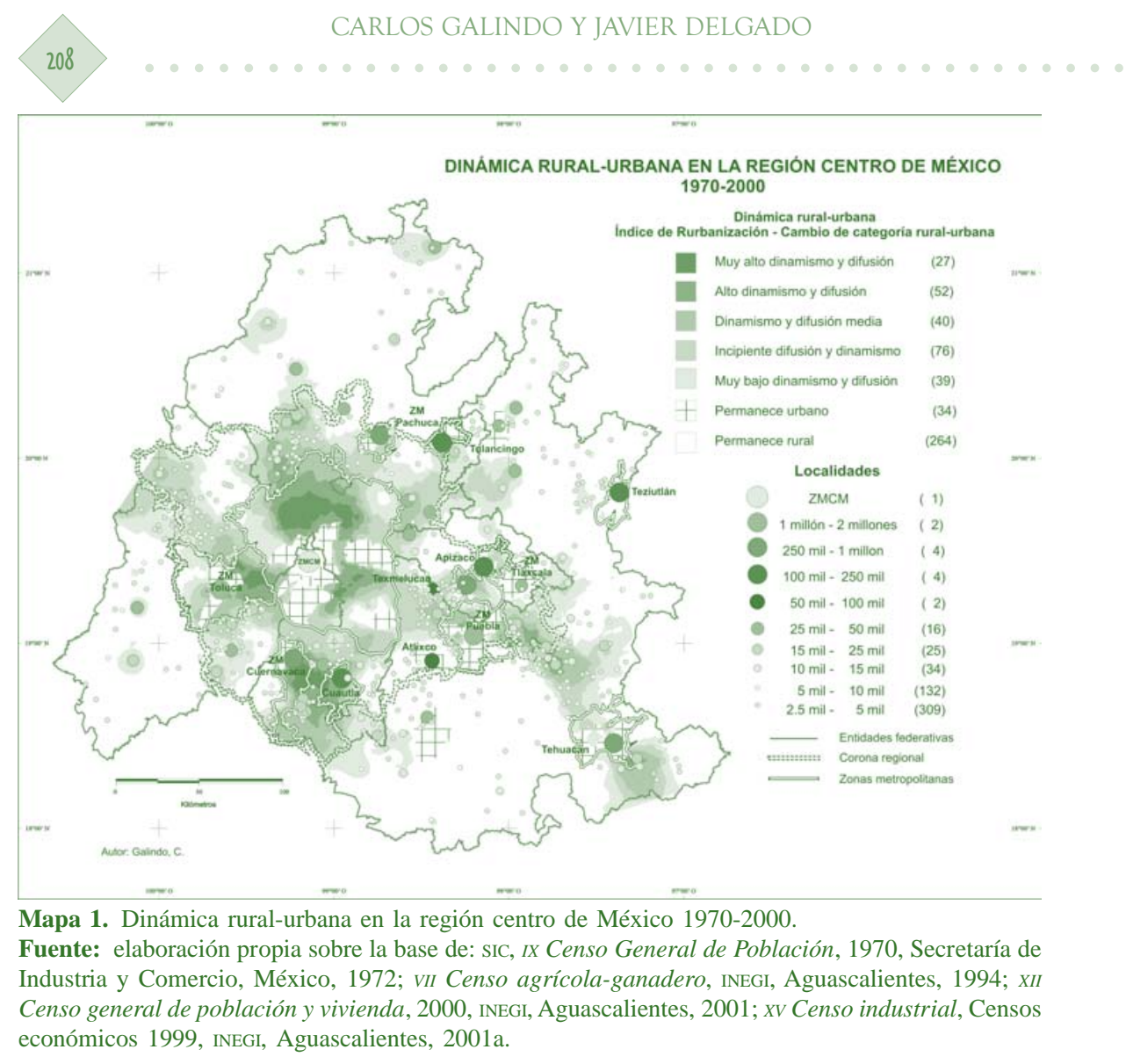

cercanos de las zonas metropolitanas de las ciudades de México, Toluca, Puebla y Cuernavaca (Mapa 1).

b) Aquellos municipios que obtuvieron valores de medio a alto dinamismo y difusión (y algunos muy alto) dentro de la corona y en la periferia regional, pero que no están dentro del periurbano de las principales zonas metropolitanas, se encuentran en la cercanía de ciudades pequeñas (entre 10 y 50 mil habitantes), lo que permite suponer la emergencia de agrupaciones subregionales asociadas a la actividad agroindustrial. El resultado permite suponer, primero, la aparición de nodos emergentes en municipios rururbanos y confirmar el papel que las ciudades pequeñas (como unidades articuladoras de los nuevos espacios subregionales) tienen en el proceso de urbanización difusa en el centro del país. Pero también especifica la medida en que la periferia reestructura al centro, mediante formas regionales de varios centros y no uno solo. Este rasgo de la dispersión es alentador como forma incipiente de un patrón no concentrado. Es probable, sin embargo, que con el influjo del mercado de suelo, la concentración termine reproduciéndose en alguno de los centros emergentes. En suma, la corona 
regional, primer contorno no conurbado de la ZMCM, se consolida como el de mayor dinamismo de toda la región Centro (Delgado, 2003).

c) Por su parte, los municipios que registraron los valores de difusión y dinamismo incipientes o muy bajos, coinciden espacialmente con la localización de ciudades de entre 2.5 a 15 mil habitantes. Como rasgo diferencial, el rango incipiente se asocia a agrupamientos de localidades menores, mientras que el muy bajo se asocia a aisladas. Esa diferencia puede explicarse por el hecho de que determinado agrupamiento de localidades, a pesar de tener muy baja jerarquía dentro de la red urbano-regional, tiene mayores posibilidades de formar parte de la dinámica económica regional dentro del proceso de urbanización difusa, siempre que esté cerca de una ciudad. ${ }^{11}$ Lo contrario sucede con aquellas localidades aisladas que permanecen en medio de grandes espacios rurales con los valores más bajos.

\section{Interfase rural-urbana y tipología de espacios periurbanos}

Una vez cartografiados, al sobreponer los mayores valores de la interfase por municipio con el sistema de ciudades por rango, destaca la formación de varias agrupaciones subregionales en la corona regional. Este resultado preliminar deberá someterse a análisis posteriores para establecer la delimitación precisa de cada agrupamiento, lo que rebasa el alcance del presente trabajo y no afecta los resultados obtenidos para el valor de la interfase. Dichas agrupaciones pueden ser útiles para estudios posteriores a escala subregional. En síntesis, se observan las siguientes agrupaciones subregionales (Cuadro 8).

Con la intención de concluir la propuesta metodológica - y a reserva de estudios locales y verificación en campo-, proponemos la siguiente tipología de agrupaciones extraídas ex post y que intenta abstraer sus características socioespaciales. Se reconocen así al menos cinco tipos de agrupaciones.

\section{Articulación de dos o más lugares centrales con interfase}

La proximidad geográfica entre dos lugares centrales no conurbados impulsa la formación de un espacio de contacto entre ellos, de interfase, caracterizado por actividades todavía rurales, en donde los usos del suelo comienzan a ser sustituidos por usos urbanos o subsisten indistintamente, derivando en una coexistencia de sus atributos socioespaciales (rurbanización).

11 No obstante, es preciso reconocer que la distancia no determina completamente la intensidad de los flujos tanto de población, como comerciales. 
Cuadro 8

Agrupaciones subregionales en espacios de interfase rural-urbana

\begin{tabular}{|c|c|c|}
\hline Entidad & En la corona & En la periferia \\
\hline Hidalgo & $\begin{array}{l}\text { Tepejí de Ocampo - San Miguel Vindho - San Marcos - } \\
\text { Tula de Allende - El Llano - Tlaxcoapan - Tezontepec - } \\
\text { Mixquiahuala - Progreso - Jilotepec (México) - } \\
\text { Ixmiquilpan (periferia) } \\
\text { - Apan - Tepeapulco - Fray Bernardino de Sahagún - } \\
\text { Zacatlán (Puebla/periferia) } \\
\varnothing \text { Actopan }\end{array}$ & $\begin{array}{l}\text { o Huejutla de Reyes } \\
\text { o Zacuatilpan } \\
\text { o Zimapán }\end{array}$ \\
\hline $\begin{array}{l}\text { Estado } \\
\text { de }\end{array}$ & $\begin{array}{l}\text { - Xalatlaco - Capulhuac de Mirafuentes - Santiago } \\
\text { Tianguistenco - Tenengo de Arista - Tenancingo - }\end{array}$ & o Valle de Bravo \\
\hline México & $\begin{array}{l}\text { - San Pedro los Baños - Atlacomulco - Temascalcingo } \\
\text { o Ixtapan de la Sal }\end{array}$ & o Tejupilco \\
\hline Morelos & $\begin{array}{l}\text { - Santa Rosa Treinta - Zacatepec de Hidalgo - Jojutla - } \\
\text { Tlaquiltenango }\end{array}$ & $\begin{array}{l}\text { - Tepalcingo-Axochiapan- } \\
\text { Chiautla de Tapia (Puebla) }\end{array}$ \\
\hline Puebla & $\begin{array}{l}\text { - Huachinango - Xicotepec } \\
\text { - Tepeaca - Acatzingo - Los Reyes de Juárez (periferia) - } \\
\text { San Salvador Huixcolotla (periferia) - Tecamachalco } \\
\text { (periferia) - Palmarito Tochapan (periferia) } \\
\text { - Ajalpan - San Sebastián Zinacatepec - San Gabriel Chilac } \\
\text { - Izúcar de Matamoros - Atencingo } \\
\text { - San Rafael Tlanalapan - Santa María Moyotzingo - } \\
\text { Santa Ana Xalmimilulco - Huejotzingo }\end{array}$ & $\begin{array}{l}\text { o Ciudad de Chignahuapan } \\
\text { o Ciudad Serdán }\end{array}$ \\
\hline Tlaxcala & $\mathrm{C}_{i}$ & \\
\hline
\end{tabular}

- Agrupación subregional. Ø Nodo conector entre agrupación subregional y zona metropolitana. o Nodo aislado

En el caso de la región Centro - un ejemplo de este tipo lo integran la ZMCM y Pachuca - en donde la interrelación mediante un espacio de interfase, en el cual tienen lugar procesos de rurbanización es evidente (Gráfica 6).

Conexión de un lugar central fuerte con un nodo secundario sobre un solo espacio de interfase

Las áreas de difusión intraregional generadas por un lugar central consolidado y otro de menor jerarquía se sobreponen en un espacio de interfase que conecta y circunda ambos nodos. Este tipo socioespacial se presenta en los binomios de Puebla-Tlaxcala y Cuernavaca-Cuautla, los primeros como los lugares centrales consolidados y los segundos como los nodos secundarios, conectados mediante un vasto territorio rururbano.

Sus rasgos distintivos son la actividad agrícola intensiva y fungir como espacio suburbano de los nodos centrales. La población ya no está sujeta a una actividad 

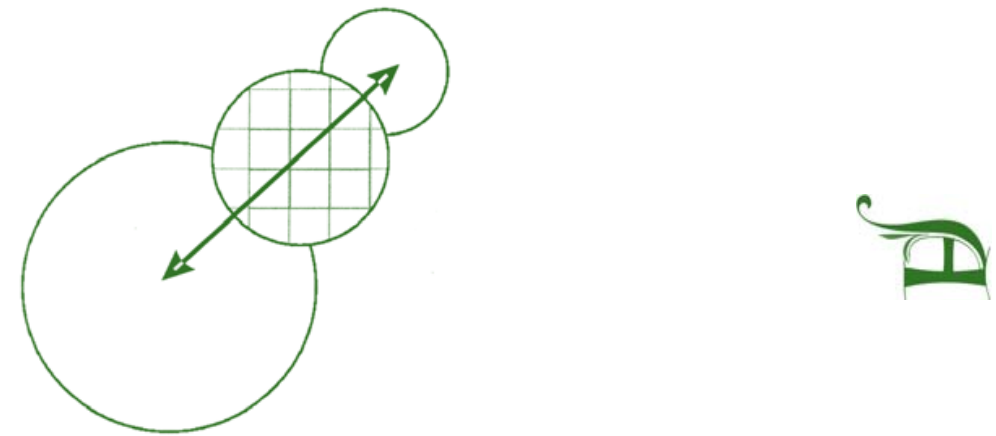

Gráfica 6. Articulación entre dos o más lugares centrales con interfase.

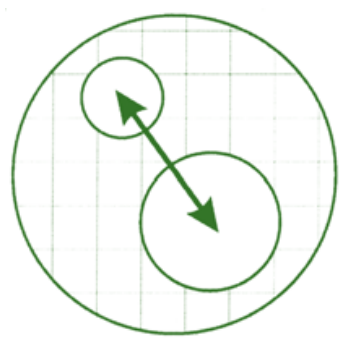

Gráfica 7. Conexión de un lugar central fuerte con un nodo secundario sobre espacio de interfase.

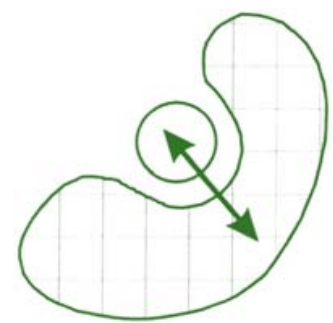

Gráfica 8. Lugar central con influencia sobre su periurbano cercano.

económica única (agricultura), sino que se diversifica en el sector secundario (maquila) o en el terciario (servicios de baja calificación) (Gráfica 7).

Lugar central con influencia sobre su periurbano cercano

Aquí destaca la presencia de numerosas localidades rurales que pueden formar agrupaciones subregionales dispersas como sucede con el extenso sector sureste de Toluca, en el Estado de México. Este tipo se presenta en los casos de un lugar central que ya expresa su influencia sobre el periurbano cercano, pero se encuentra separado de otros lugares centrales dentro de la región (Gráfica 8). 


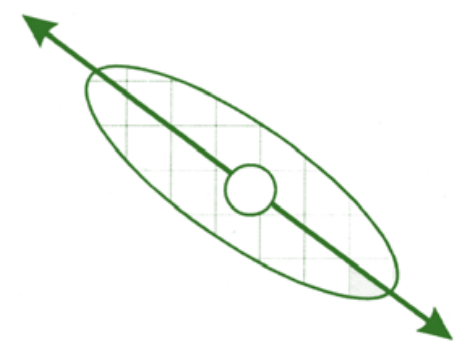

Gráfica 9. Nodo conector sobre corredor regional.

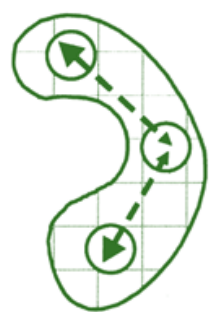

Gráfica 10. Aglomeración de nodos secundarios sobre espacio de interfase.

Nodo conector sobre corredor regional

La ubicación estratégica de un lugar central sobre un eje regional de comunicación, favorece la formación de un nodo conector entre dos o más lugares centrales, que aprovecha la intensidad de los flujos y vínculos interregionales. El caso paradigmático de este tipo es Tehuacán, que funge como conector entre las ciudades de Puebla y Oaxaca sobre un corredor regional que va desde Guadalajara en el Bajío y pasa por Querétaro y la zMCM (Gráfica 9).

Aglomeración de nodos secundarios sobre espacio de interfase

El distanciamiento geográfico respecto de un lugar central fuerte, pero la cercanía entre sí de un conjunto de nodos urbanos de menor jerarquía, conduce a la formación de agrupaciones subregionales interconectadas mediante un espacio de interfase, donde todavía ninguno de los nodos es hegemónico. Caracterizado por una actividad agropecuaria en la que la sinergia de caracteres urbanos y rurales es ya considerable, lo que da lugar a la formación de un enclave rururbano (Gráfica 10).

Los casos paradigmáticos de las numerosas agrupaciones subregionales se encuentran en Hidalgo, en el vasto espacio rururbano del valle del río Tula y otras dos en Puebla, una al oriente y la tercera y última al norte (véase Cuadro 8). 


\section{Conclusiones preliminares}

Heterogeneidad y dinamismo de la corona regional

A pesar de que se trata de la primera exploración para medir la dinámica ruralurbana en espacios periurbanos de grandes metrópolis, sus resultados permiten identificar esos espacios rurales peculiares y su coexistencia con la dinámica urbana anteriormente detectada mediante la medición de la corona regional.

Visto así, lo novedoso de esos espacios de mayor dinamismo rural es que se presentan indistintamente en el espacio periurbano de zonas metropolitanas, ciudades medias o aun pequeñas. Destaca también la variedad de los tipos específicos de conformación territorial que están adoptando esos agrupamientos, muy lejos de la supuesta dicotomía urbano-rural. ¿Terminarán esos espacios por devenir finalmente urbanos?, ¿tendremos ante nosotros formas inéditas de organizar el territorio, si bien todavía bajo el comando de un lugar central fuerte?, ¿esta nueva forma - heterogénea, diversa, muy dinámica一, representará una ventaja más que desventaja ante las conocidas limitaciones de la anterior concentración? En el nivel de desarrollo del presente ensayo no podemos dar respuestas definitivas, la exploración aquí mostrada tiene la finalidad de incentivar nuevas formas de interrogar al territorio.

Surgimiento de nodos en la periferia regional asociados a procesos fuera de la región Centro

De manera similar, los nodos urbanos emergentes en la periferia regional, detectados desde el primer ejercicio de la corona regional en 1995, corresponden también a los rurales en el espacio periurbano cercano a esos nodos, los cuales, suponemos, están más vinculados con otros lugares centrales fuera de la región Centro, dado que su vinculación con los nodos intraregionales no parece clara.

\section{Alcances y limitaciones de la metodología propuesta}

La metodología propuesta es un primer intento formal por superar los planteamientos del paradigma tradicional de oposición campo-ciudad: constituye una forma novedosa de abordar simultáneamente procesos que se ubican entre las escalas urbana y regional, subraya el papel del territorio como factor explicativo de los procesos analizados y es factible que pueda aplicarse a un buen número de otras interrelaciones significativas entre los ámbitos rural y urbano, incluyendo el análisis de los recursos naturales ubicados en el periurbano.

En cambio, es deseable la incorporación de otras variables más representativas de la rurbanización si estuviesen disponibles. Por ejemplo, la extensión de la segunda 
residencia y de sus usuarios (que en otros países está disponible censalmente), los desplazamientos de la población entre y desde de esos vastos espacios periurba-nos, los motivos y frecuencia de sus desplazamientos y, sobre todo, no limitarlos a la adscripción metropolitana como sucede con la muestra del último censo. Con este ejemplo, es claro cómo la delimitación metropolitana es una camisa de fuerza de la dimensión periurbana.

Nunca antes como ahora, lo urbano se vincula con lo rural. Campo y ciudad no son polos opuestos, sino que coexisten en tiempo y espacio. Los esfuerzos de conceptuación teórica, aún incipientes, deberán profundizarse. Esperamos que los agrupamientos subregionales aquí identificados tengan algún sentido para quienes, desde el ámbito local, analizan esos espacios peculiares. La comprobación mediante trabajo de campo en éstos es ya una labor impostergable.

\section{Bibliografía}

Aguilar, Adrián, "Las megaciudades y las periferias expandidas”, en EURE, vol. 28, núm. 85, Santiago de Chile, 2002, pp. 121-149.

Alonso, William, Location and land use, Boston, mit Pres, 1964.

Arias, Patricia, "Nueva ruralidad antropólogos y geógrafos frente al campo hoy", en Héctor Ávila (coordinador), Lo urbano-rural, ¿nuevas expresiones territoriales, México, Centro Regional de Investigaciones Multidisciplinarias, unAM, Cuernavaca, 2005, pp. 123-159.

Ávila, Héctor, "Ideas y planteamientos teóricos sobre los territorios periurbanos, Las relaciones campo-ciudad en algunos países de Europa y América", en Investigaciones Geográficas, Boletín del Instituto de Geografía, núm. 45, México, Instituto de Geografía, unAm, 2001, pp. 108-127.

, "La agricultura en las ciudades y su periferia un enfoque desde la Geografía", en Investigaciones Geográficas, Boletín del Instituto de Geografia, núm. 53, Instituto de Geografía, México, unAm, 2004, pp. 98-121.

Banzo, Mayte, "Del espacio al modo de vida. La cuestión periurbana en Europa Occidental los casos de Francia y España”, en H. Ávila (coordinador), Lo urbano-rural, ¿nuevas expresiones territoriales, Cuernavaca, México, Centro Regional de Investigaciones Multidisciplinarias, UNAM, 2005, pp. 207-241.

Benko, George y Alan Lipietz, "El nuevo debate regional”, en George Benko y Alan Lipietz (compilador), Las regiones que ganan, Madrid, Ediciones Alfonso El Magnánimo, 1994, pp. 19-36.

Borja, Jordi y Manuel Castells, Local y Global, La gestión de las ciudades en la era de la información, Madrid, Centro de la Naciones Unidas para los Asentamientos Humanos, Taurus, 1999.

Boudeville, Jacques, Les espaces economiques, Paris Presses Universitaires de France, 1961.

Camposortega, Sergio, "Evolución y tendencias demográficas de la ZMCM", en CONAPO, $L a$ Zona Metropolitana de la Ciudad de México. Problemática actual y perspectivas demográficas y urbanas, México, Consejo Nacional de Población, 1992, pp. 3-15.

Claval, Paul, Geografia Económica, Barcelona, Oikos-tau, 1980.

Cochrane, Steven y Daniel Vining Jr., "Recent trends in migration between core y peripheral regions in developed y advanced developing countries", en International Regional Science Review, vol. 11, núm. 1, 1988, pp. 215-243.

Cromley, Robert, "A comparison of optimal classification strategies for choropletic displays of spatially aggregated data", en International Journal of Geographical Information Systems, vol. 10, núm. 4, 1996, pp. 405-424.

Cruz, María. Soledad, "Procesos urbanos y 'ruralidad' en la periferia de la Zona Metropolitana de la Ciudad de México", en Estudios Demográficos y Urbanos, vol., 17, 
núm. 1, El México, Colegio de México, 2002, pp. 39-76.

De Blij, Harm, Geography Regions y Concepts, John Wiley y Sons, Inc, Nuva York, 1981.

Dear, Michael y Steven Flusty, "Postmodern Urbanism", en Dear, Michael y Steven Flusty (editores), The spaces of postmodernity, Readings in Human Geography, Oxford, Blackwell Publishers, 2002, pp. 216-234.

Delgado, Javier, "La ciudad en transición", en Delgado, J. y D. Villareal (coordinadores), Cambios territoriales en México, Exploraciones recientes, México, Universidad Autónoma Metropolitana-Xochimilco y Centro de Ecodesarrollo, 1991, pp. 217-229.

, "La nueva ruralidad en México", en Investigaciones Geográficas, Boletín del Instituto de Geografia, núm. 39, México, Instituto de Geografía, unAM, 1999, pp. 82-93.

, "La urbanización difusa, arquetipo territorial de la ciudad-región", en Sociológica, año 18, núm. 51, enero-abril, México, 2003, pp. 13-48.

, "Transición rural-urbana y oposición campo-ciudad", en A. G. Aguilar Martínez, (coordnador), Urbanización, cambio tecnológico y costo social, El caso de la Región Centro de México, México, Instituto de Geografia de la Universidad Nacional Autónoma de México/Consejo Nacional de Ciencia y Tecnología/Editorial Miguel Ángel Porrua, 2003a, pp. 73-118.

Delgado, Javier, Adriana Larralde y Carlos Anzaldo, "La corona regional de la ciudad de México, Primer anillo exterior en formación", en Javier Delgado y Blanca Ramírez (coordinadores), Transiciones, Territorio y cultura en la Ciudad de México, México, tomo 1, Universidad Autónoma Metropolitana/Plaza y Valdez, 1999, pp. 171194.

-, Gabriela Ángeles y Carlos Galindo, ’La interfase sociedad-naturaleza, un enfoque territorial a la fragmentación espacial de la urbanización difusa", en ponencia presentada en el Encuentro Anual de la Unión Geográfica Internacional (IGU), Eslovenia, 2003.

y Naxhelli Ruiz, "Modelos urbanos y regionales. Hacia una aglomeración multiescala de ciudades", en A. Ziccardi (coordinador), Regiones del Siglo XX, México, Instituto Mora, 2004.

Dematteis, Giusseppe, "Suburbanización y periurbanización, Ciudades anglosajonas y ciudades latinas", en Javier Monclús (editor), La ciudad dispersa suburbaniza- ción y nuevas periferias, Barcelona, Centre de Cultura Contemporania de Barcelona, 1998, pp. 17-33

Flores, Ernesto, Tratado de economía agrícola, México, Fondo de Cultura Económica, 1961.

Galindo, Carlos "Urbanización difusa, territorio y rearticulación urbano-regional en el centro de México", tesis de Maestría en Geografía, México, Facultad de Filosofía y Letras, UNAM, 2006.

Garza, Gustavo, Evolución de la Ciudad de México en el siglo $X X$, México, Cuadernos de Trabajo del Centro de Estudios Demográficos y de Desarrollo Urbano, El Colegio de México, 1992.

Giménez, Gilberto, "Territorio, cultura e identidades, La región socio-cultural", en Rocío Rosales, Globalización y regiones en México, Programa Universitario de Estudios sobre la Ciudad, México, Facultad de Ciencias Políticas y Sociales, unAm/Miguel Ángel Porrúa, 2000, pp. 19-52.

Gutiérrez-Puebla, Javier, La ciudad y la organización regional, Madrid, Editorial Cincel, 1992.

Graizbord, Boris y Gallardo, Georgina,"La ciudad de México aislada (La centralización de las comunicaciones telefónicas)", en Estudios Demográficos y Urbanos, vol. 1, núm. 1, México, El Colegio de México, 1986, pp. 125-131.

Harris, Chaucy, "The nature of cities y Urban Geography in the last half century", Urban Geography, January-February, 14, vol. 18, núm. 1, 1997, pp. 15-35.

Hiernaux, Daniel, "Las nuevas formas urbanas y reestructuración del mundo rural", en P. Torres Lima (compilador), Procesos metropolitanos y agricultura urbana, México, Universidad Autónoma Metropolitana, Unidad Xochimilco/Organización de las Naciones Unidades para la Agricultura y la Alimentación, 2000, pp. 31-41.

INEGI, XII Censo Industrial, Censos Económicos 1986, INEGI, Aguascalientes, 1989.

_, VII Censo Agrícola-Ganadero, INEGI, Aguascalientes, 1994.

- XII Censo General de Población y Vivienda, 2000, INEGI, Aguascalientes, 2001. , XV Censo Industrial, Censos Económicos 1999, INEGI, Aguascalientes, 2001a. , XVI Censo Industrial, Censos Económicos 2004, INEGI, Aguascalientes, 2006.

Negrete, Maria Eugenia, "Desconcentración poblacional en la región Centro de México", en Estudios Demográficos y Urbanos, vol. 14, núm. 2, México, El Colegio de México, 1999 , pp. 313-352. 
Sociológica, año 18, núm. 51, enero-abril, México, 2003a pp. 99-127.

"Zonas Metropolitanas de México en 2000 conformación territorial y movilidad de la población ocupada", en Estudios Demográficos y Urbanos, vol. 18, núm. 3, México, El Colegio de México, 2003b, pp. 461-507. Sobrino, Luis Jaime, "Tendencias de la urbanización mexicana hacia finales de siglo" en Estudios Demográficos y Urbanos, vol. 1, núm. 11, El Colegio de México, 1996, pp. 101-137.

Suárez, Manuel y Javier Delgado, "La expansión urbana probable de la Ciudad de México. Un escenario pesimista y dos alternativos para el año 2020", en Estudios Demográficos y Urbanos, El Colegio de México, México, 2006a (en prensa).

Suárez, Manuel y Javier Delgado, "Estructura y eficiencia urbana, accesibilidad a empleos, localización residencial e ingreso en la ZMCM, 1990-2000“ en Espacio, Sociedad y Territorio, El Colegio Mexiquense, 2006b (en prensa).

Torres Lima, Pablo, "Sustentabilidad y agricultura urbana”, en Pablo Torres Lima (compilador), Procesos metropolitanos y agricultura urbana, Universidad Autónoma Metropolitana, Unidad Xochimilco y Organización de las Naciones Unidades para la Agricultura y la Alimentación, México, 2000, pp. 9-15.

Unikel, Luis, Cresencio Ruiz y Gustavo Garza, El desarrollo urbano de México diagnostico e implicaciones futuras, México, El Colegio de México, 1978.

Vining, Daniel y Robert Pallone, "Migration between core y peripheral regions a description of the patterns in 22 countries", en Geoforum, vol. 13, núm. 4, 1982, pp. 339-410. 